This item was submitted to Loughborough's Research Repository by the author.

Items in Figshare are protected by copyright, with all rights reserved, unless otherwise indicated.

\title{
Thermohydrodynamics of lubricant flow with carbon nanoparticles in tribological contacts
}

PLEASE CITE THE PUBLISHED VERSION

http://dx.doi.org/10.1016/j.triboint.2016.12.048

PUBLISHER

(C) Elsevier

VERSION

AM (Accepted Manuscript)

\section{PUBLISHER STATEMENT}

This work is made available according to the conditions of the Creative Commons Attribution-NonCommercialNoDerivatives 4.0 International (CC BY-NC-ND 4.0) licence. Full details of this licence are available at: https://creativecommons.org/licenses/by-nc-nd/4.0/

\section{LICENCE}

CC BY-NC-ND 4.0

\section{REPOSITORY RECORD}

Shahmohamadi, Hamed, Ramin Rahmani, Homer Rahnejat, Colin Garner, and Nikolaos Balodimos. 2016. "Thermohydrodynamics of Lubricant Flow with Carbon Nanoparticles in Tribological Contacts". Loughborough University. https://hdl.handle.net/2134/23877. 


\title{
Thermohydrodynamics of lubricant flow with carbon nanoparticles in tribological contacts
}

\author{
H. Shahmohamadi ${ }^{1}$, R. Rahmani ${ }^{1}$, H. Rahnejat ${ }^{1}$, C.P. Garner ${ }^{1}$, N. Balodimos ${ }^{2}$ \\ ${ }^{1}$ Wolfson School of Mechanical, Electrical and Manufacturing Engineering, Loughborough \\ University, Leicestershire, UK \\ ${ }^{2}$ Specialist Lubricants Ltd., Livingston, UK
}

\begin{abstract}
This paper deals with the tribological performance of carbon nanoparticles dispersed in polyalphaolefin PAO6 oil. Stribeck curves are obtained under various operating conditions, using a fully instrumented pin-on-disc tribometer under controlled conditions. A detailed multi-physics thermal fluid flow model with Lagrangian low concentration discrete solid phase and Eulerian multi-phase fluid with cavitation represented by modified RayleighPlesset and vapour transport equation is presented. The numerical predictions under identical conditions to the experiments show good conformance with the measurements, and provide a fundamental understanding of the role of nanoparticles. Results show improved heat transfer from the contact with the presence of nanoparticles even at low levels of concentration. The analysis shows that this leads to higher lubricant viscosity, load carrying capacity and reduced friction. Furthermore, a resulting small region of cavitation at low volume fraction does not unduly affect the enhanced heat transfer of nanoparticles. This combined experimentation and detailed numerical analysis has not hitherto been reported in literature.
\end{abstract}

Keywords: Multiphase flow; Lagrangian model; Cavitation; Nanolubricant (Nanofluid);

Sliding contact; Friction; Heat transfer

\section{Nomenclature}
$A$
Apparent contact area
$C_{c} \quad$ Cunningham slip correction factor
$d_{p} \quad$ Diameter of nanoparticles
$E^{*} \quad$ Young's modulus of elasticity
$f_{b} \quad$ Boundary friction 


\begin{tabular}{|c|c|}
\hline$f_{t}$ & Total friction \\
\hline$f_{v}$ & Viscous friction \\
\hline$H$ & Enthalpy \\
\hline$h_{m}$ & Minimum film thickness \\
\hline$h$ & Heat transfer coefficient \\
\hline$k$ & Oil thermal conductivity \\
\hline$p_{a t m}$ & Atmospheric pressure \\
\hline$p_{\text {sat }}$ & Cavitation (vaporisation) pressure \\
\hline$p_{h}$ & Hydrodynamic pressure \\
\hline $\operatorname{Re}$ & Reynolds number \\
\hline$S$ & Integration variable \\
\hline$S_{0}$ & Temperature-viscosity index \\
\hline$t$ & Time \\
\hline$\vec{V}$ & Velocity vector \\
\hline$u, v$ & Components of velocity in Cartesian coordinates \\
\hline$x, y$ & Cartesian coordinates \\
\hline$Z$ & Pressure-viscosity index \\
\hline
\end{tabular}

\section{Greek symbols}

$\alpha_{0}, \beta_{0} \quad$ pressure/temperature-viscosity coefficient

$\delta_{i j} \quad$ Kronecker delta

$\zeta \quad$ Number of asperity peaks per unit contact area

$\eta \quad$ Oil dynamic viscosity

$\eta_{0} \quad$ Oil dynamic viscosity at atmospheric pressure

$\kappa \quad$ Average asperity tip radius 
$\lambda \quad$ Mean molecular free path

$\lambda_{s} \quad$ Stribeck’s film ratio parameter

$\varsigma \quad$ Pressure coefficient for boundary shear strength of asperities

$\rho \quad$ Oil density

$\rho_{p} \quad$ Nanoparticle density

$\rho_{0} \quad$ Oil density at atmospheric pressure

$\tau \quad$ Shear stress

$\tau_{0} \quad$ Eyring shear stress

$\Gamma \quad$ Diffusion coefficient

T Temperature

\section{Abbreviations}

CFD Computational fluid dynamics

MSDS Material Safety Data Sheets

PAO Poly-alpha-olefin

RMS Root Mean Square

RPM Revolutions per Minute

\section{Introduction}

Nanofluids are essentially stable and durable colloidal suspensions made of nanoparticles with sizes usually in the range 1-120 nm, dispersed in the base fluid. When the base fluid is an oil, the resultant colloidal suspension is referred to as a nano-lubricant or nano-oil. The mechanisms involved in reducing friction and wear are mostly dependent on the characteristics of nanoparticles including size, shape, and volumetric concentration. For tribological applications, the nanoparticles should generally have lower friction coefficient, high dispersion properties, act as extreme pressure (EP) agents, and enhance thermal conductivity. At intense thermal loading and heat fluxes, the nanofluids assume an important 
role in heat transfer. To produce nanolubricants, nanoparticles are dispersed in the base fluid using mechanical stirring and ultrasonic agitation to form a stable suspension.

Nanoparticles made of different materials, added to various types of lubricants, have been investigated in the recent past in order to find their influence in reducing friction and wear in various contacts. Greenberg et al [1] investigated the effect of nested inorganic fullerene-like (IF) metal dichalcogenide $\mathrm{WS}_{2}$ nanoparticles, added to various base oils in reducing friction under different regimes of lubrication. They used three different tribometers including a flaton-flat, roller-on-rib and ball-on-flat configurations. They observed a $50 \%$ reduction in the coefficient of friction in mixed regime of lubrication. They presented their results for coefficient of friction in the form of a Stribeck curve covering all regimes of lubrication from hydrodynamic to boundary. They hypothesised that the mechanism through which a reduction in the coefficient of friction occurs is the formation of tribo-films of $\mathrm{WS}_{2}$. Wu et al [2] investigated the effect of $\mathrm{CuO}, \mathrm{TiO}_{2}$ and nano-diamond nanoparticles as additives in a base oil as well as in an API-SF engine oil, in reducing friction and wear. They used a TE-77 reciprocating sliding tribometer. Reductions of $18.4 \%$ and $5.8 \%$ were observed by adding the $\mathrm{CuO}$ nanoparticles to the engine lubricant and the base oil respectively. However, they observed relatively small reduction in friction when the nano-diamond particles were used.

The friction reduction properties of $\mathrm{CuO}$ nanoparticles added to polyalphaolefin PAO6 on coating of NiCrBSi were investigated by Battez et al [3]. The reduction in friction because of nanoparticles was attributed to two main parameters; firstly the nanoparticles acting as thirdbody nano-bearings, and secondly formation of tribofilms through their adsorption to the coating material.

Using a disc-on-disc tribometer, Lee et al [4] and $\mathrm{Ku}$ et al [5] employed fullerence (C60) nanoparticles added to mineral oils to investigate their tribological performance. Ku et al [5] used a volume fraction of $0.1 \%$, whilst Lee et al [4] changed the volume fraction from $0.01 \%$ to $0.5 \%$. They showed their results for coefficient of friction in the form of Stribeck curves. Lee et al [4] found that the volume fraction (VF) of fullerence was an important factor in reducing the coefficient of friction, whilst Ku et al [5] observed a more effective nanoparticle performance with lower viscosity base oils. Furthermore, using a disc-on-disc tribometer, Lee et al [6] produced Stribeck curves to study the performance of graphite nano-lubricants. They found that the nano-lubricant with $0.5 \% \mathrm{VF}$ of graphite produced the least coefficient of friction. 
Wan et al [7] investigated the use of boron nitride nanoparticles in commercial lubricating oil (SE 15W-40). Optimal size and concentration of particles were found to be $\sim 120 \mathrm{~nm}$ and 0.1 wt $\%$, reducing friction by as much as $77 \%$ with respect to the base oil. Oleic acid was used as a dispersant to ensure suspension stability. A high shear homogeniser was used for dispersing the particles. However, Wan et al [7] did not observe any difference between viscosity of the base oil and that with the inclusion of nano-lubricants with different levels of concentration.

The above studies and many other similar investigations have shown the advantages of using nanoparticle in reducing friction in various contacts, particularly using various tribometers. However, they do not provide an in-depth analysis of the underlying mechanisms involved in flow dynamics of the nanoparticles within the bulk lubricant. It is important to ascertain in a fundamental manner the key roles which particle shape, size and concentration play through use of multi-phase flow dynamics.

There is a relatively a large volume of reported analysis, but confined to the effectiveness of nanoparticles in the promotion of additional heat transfer to or from fluid flows. A review of these studies can be found in Godson et al [8] or Kaymar et al [9]. There are no reported analyses of nanoparticle performance in fluid flows through tribological conjunctions. Therefore, the current study includes a fluid dynamics analysis, where the nanofluid flow is defined as a combination of a continuous liquid phase and a dispersed solid particle phase. The Eulerian-Lagrangian approach is used in the solution of Navier-Stokes equations. The Eulerian description assumes a continuum medium for the fluid phase with conservation laws for the liquid phase, whilst detailed particle motion is described through use of Lagrangian dynamics. The particle-particle interaction forces are neglected in the current study due to the relatively low concentration of the nanoparticles in the base oil. The result of analysis conforms well to the experimental measurements using a pin-on-disc tribometer under a broad range of regimes of lubrication. Such an approach has not hitherto been reported in literature.

\section{Experimental Measurements}

An in-house pin-on-disc tribometer is used to simulate the flow of a nano-lubricant in a typical converging-diverging sliding contact. Furthermore, the tribometric tests provide means of validation for numerical predictive method.

\subsection{Preparing the nano-lubricant samples}


The base oil used is PAO6 with the properties listed in Table1. Carbon nanoparticles with $0.02 \%$ volume fraction are suspended in the base oil with mechanical stirring. The main reason for the $0.02 \%$ solution is that this particular concentration provides a very stable mixture, not encountering any separation problems. Upon examination of samples with various concentrations it was found that solutions produced with this particular concentration of particles remained stable for well over a year.

Diamond nanoparticles (DND) were initially produced in the USSR during the 1960s by detonation of carbon containing explosives, usually with a negative oxygen balance. In recent years, these particles have become the topic of extensive studies due to their excellent mechanical and optical properties, low toxicity compared with other carbon nanoparticles, high surface area and tuneable surface structure. Owing to these characteristics their use for a number of different applications is being researched, including their use as a lubricant additive.

The blending process is performed using a standard mechanical mixer. The produced mixtures also include a relatively small amount of molybdenum phosphorodithioate (no exceeding $0.1 \%$ wt.). Molybdenum phosphorodithioate is present as an additive that can further enhance the performance of the end product through synergistic effect.

Table1: Properties of the base oil (PAO6)

\begin{tabular}{|c|c|c|}
\hline Parameter & Value & Unit \\
\hline Viscosity at $40^{\circ} \mathrm{C}$ & 30 & $\mathrm{mPa} . \mathrm{s}$ \\
\hline Viscosity at $100^{\circ} \mathrm{C}$ & 6 & $\mathrm{mPa} . \mathrm{s}$ \\
\hline Density & 826 & $\mathrm{~kg} / \mathrm{m}^{3}$ \\
\hline Thermal conductivity & 0.144 & $\mathrm{~W} /(\mathrm{mK})$ \\
\hline Specific heat capacity & $2.303 \times 10^{3}$ & $\mathrm{~J} /(\mathrm{kgK})$ \\
\hline
\end{tabular}

Lubricant viscosity was measured using Brookfield Rheometer DV-II+ Pro, equipped with a $\mathrm{RV}-1$ spindle. The data related to the thermal properties of the lubricant, including thermal conductivity and specific heat capacity were extracted from the Material Safety Data Sheets (MSDS). The thermal properties of the lubricant including thermal conductivity and specific heat capacity were also measured. 


\subsection{Preparing pin and disc samples}

The pin and disc samples are both made out of steel. The disc was turned on a lathe and the pin was made using a milling machine. Both pin and disc were polished using a PM5 (Precision Lapping and Polishing System). A scaled rotating fixture was used to create a symmetric parabolic wedge profile on the pin sample to create an inlet wedge for the entrainment of the lubricant into the contact through hydrodynamic pressure rise. Surface profile of the pin sample was measured using an Alicona Infinite Focus microscope with the vertical resolution of $\pm 1 \mathrm{~nm}$ and horizontal resolution of $0.175 \mu \mathrm{m}$. Figure 1 shows the measured pin profile, as well as the fitted curve to the measured profile, which is used in the numerical analysis. The effect of surface roughness on the tribological performance of the contact particularly in boundary regime of lubrication is included through use of measured roughness parameter and by removing the surface form from the measured data.

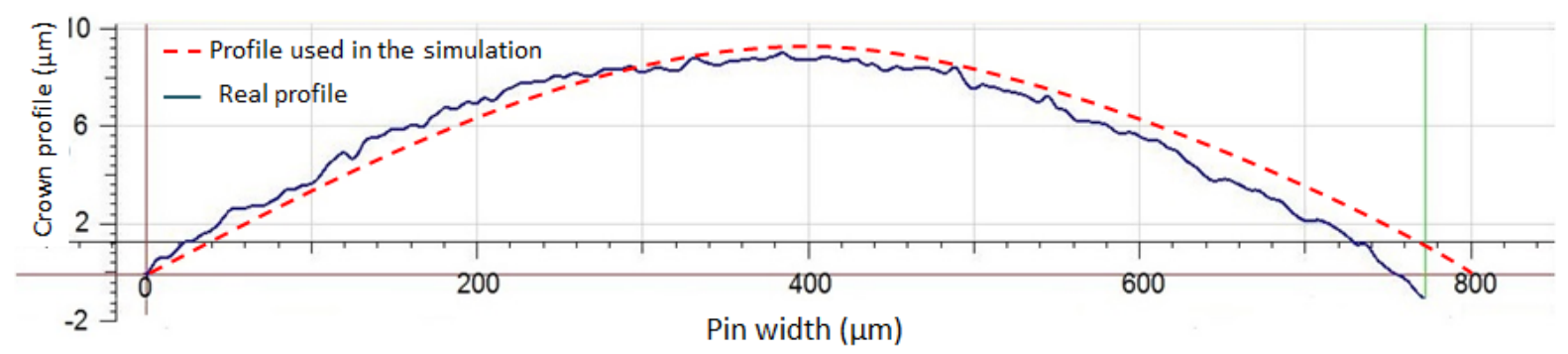

Figure1: Pin sample profile

Surface topographical data for both the pin and the disc samples are listed in Table 2.

Table 2: Surface topography parameters for both pin and disc samples

\begin{tabular}{|c|c|c|c|c|}
\hline Parameter & Description & Value (pin) & Value (disc) & Unit \\
\hline Sa & Average roughness & 0.104 & 0.138 & $\mu \mathrm{m}$ \\
\hline Sq & RMS roughness & 0.136 & 0.198 & $\mu \mathrm{m}$ \\
\hline Ssk & Skewness & -0.188 & 0.254 & - \\
\hline Sku & Kurtosis & 4.444 & 3.809 & - \\
\hline Spd & Peak density & 4833 & 6138 & $\mathrm{~mm}^{-2}$ \\
\hline Spc & Peak average curvature & 711 & 918 & $\mathrm{~mm}^{-1}$ \\
\hline
\end{tabular}


According to Table 2, the distribution of roughness in both the samples somewhat deviate from a normal (Gaussian) distribution. In the case of the pin sample, the valleys are slightly predominant, whilst in the case of the disc, roughness peaks are slightly predominant.

\subsection{Experimental set up and running procedure}

Rotational speeds for the disc were 50, 100, 150, 200, 320, 600, 1000 and 1100 RPM. These correspond to average contact sliding speeds of $0.2,0.4,0.6,0.8,1.3,2.4,4$ and $4.5 \mathrm{~m} / \mathrm{s}$ respectively. The applied normal load on the pin was set at 5 and $10 \mathrm{~N}$. Considering a line contact between the pin and the disc at the apex of the parabola and using Hertzian contact theory, the deflection for an elastic line contact of small, but finite width becomes [10]:

$\delta=\frac{P^{\prime}}{\pi E^{*}}\left[\ln \left(\frac{L^{2} \pi E^{*}}{2 R P^{\prime}}\right)+1\right]$

Localised deformations of 6 and $11 \mathrm{~nm}$ are predicted for the contact loads of 5 and $10 \mathrm{~N}$ respectively. This indicates that under the applied loads, no significant local elastic deformation occurs in the conjunction. This chosen range of speed and load is to ensure a full sweep of various regimes of lubrication from hydrodynamic to boundary, which are typically demonstrated in Stribeck curves.

The lubricant was supplied into the conjunction by a Harvard Apparatus 11 Elite syringe pump to ensure a continuous and controlled inlet supply rate.

\section{Numerical Model}

A Lagrangian discrete phase model (Euler-Lagrange approach) is used in the current study. In this approach, an Eulerian method is used to treat the flow field whilst a Lagrangian approach describes the motion of the discrete elements (i.e. the nanoparticles) suspended in the fluidic continuum.

\subsection{Equations of motion for the fluid phase}

The fluid phase is treated as a continuum by solving the Navier-Stokes equations [11]:

$$
\begin{aligned}
& \frac{D \rho}{D t}+\rho \vec{\nabla} \cdot \vec{V}=0 \\
& \rho \frac{D \vec{V}}{D t}=-\vec{\nabla} p+\vec{\nabla} \cdot\left(\bar{\tau}_{i j}\right)+\vec{F}
\end{aligned}
$$


where, $D / D t$ is the material covariant derivative, $\rho$ is the lubricant density, $p$ is pressure, $\vec{V}$ is the velocity vector and $\bar{\tau}_{i j}$ is the viscous shear stress tensor:

$$
\bar{\tau}_{i j}=\eta\left(\frac{\partial u_{i}}{\partial x_{j}}+\frac{\partial u_{j}}{\partial x_{i}}-\frac{2}{3} \delta_{i j} \nabla \cdot \vec{V}\right)
$$

where, $\eta$ is the lubricant viscosity $\delta_{i j}$ is the Kronecker delta.

Although negligible in this particular case, the body forces in the fluid, $\vec{F}$, are also included for the sake of generality.

\subsection{Thermal analysis}

To take into account the rise in lubricant temperature in the contact due to frictional heating, the energy equation is solved in the flow domain. Energy conservation equation in the general form can be stated as [11]:

$\rho \frac{D H}{D t}=\frac{D p}{D t}+\vec{\nabla} \cdot(k \vec{\nabla} T)+\dot{q}$

where, $H$ is the fluid enthalpy, $T$ is temperature, $k$ is the lubricant thermal conductivity, and the source term $\dot{q}$ represents the generated heat in the contact. The frictional heating originates from the viscous shear of the lubricant in the contact, as well as through the direct contact of asperity tip on the counter face surfaces (i.e. the pin and the disc) due to generated boundary friction. Therefore, the source term can be obtained by calculating the frictional power losses in the contact due to both viscous shear of the lubricant and also the direct contact of the asperities.

To evaluate the contact temperature more accurately, the heat conduction equation is solved for both the pin and the disc as:

$$
\nabla^{2} T=0
$$

In this model, the transient effects are ignored and hence the steady-state form of the governing equations for heat transfer in the sliding bodies is considered. 


\subsection{Equations of motion for the nanoparticles}

Lagrangian dynamics is used to solve the equations for the dispersed discrete phase (i.e. the nanoparticles), in which a large number of particles are tracked individually throughout the flow field. In this method, the dispersed phase can exchange momentum, mass, and energy within the fluid phase. This approach is made considerably simpler when particle-particle interactions are neglected. Considering the relatively low concentration of the nanoparticles in the studied lubricant $(0.02 \%$ by volume), this simplification is expected not to affect the results significantly. Nevertheless, a thorough analysis method may need to incorporate such interactions. Using this method, the particle trajectories are computed individually at specified intervals during the fluid phase computations.

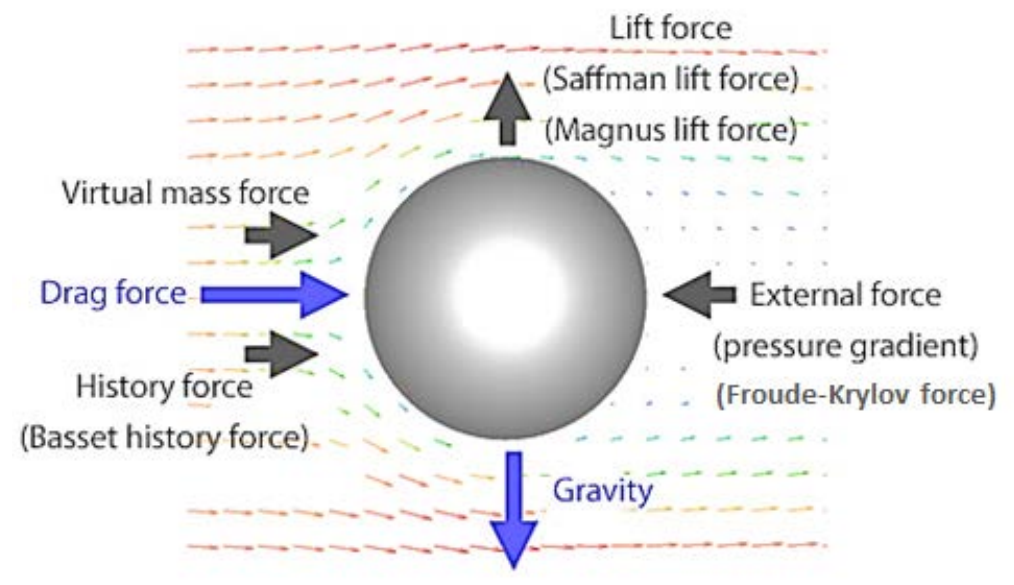

Figure 2: Forces acting on a single particle

The trajectory of a discrete phase particle is predicted by integrating the balance of forces acting upon it (figure 2), which is written in a Lagrangian frame of reference. This force balance equates the particle inertial force (Newton's second axiom). Assuming that the nanoparticles are spherical and considering that the Reynolds numbers in lubrication problems are generally low, the equation of motion for a small particle can be formulated using modified Basset-Boussinesq-Oseen equation, governing momentum transfer between the fluid and a particle as [12]:

$$
\begin{aligned}
\frac{\pi}{6} \rho_{p} d_{p}^{3} \frac{\partial \vec{V}_{p}}{\partial t} & =3 \pi \eta d_{p}\left(\vec{V}-\vec{V}_{p}\right)-\frac{\pi}{6} d_{p}^{3} \vec{\nabla} p+\frac{\pi}{12} \rho d_{p}^{3}\left(\frac{D}{D t} \vec{V}-\frac{\partial}{\partial t} \vec{V}_{p}\right)+ \\
& +\frac{3 \sqrt{\pi}}{2} \sqrt{\rho \eta} d_{p}^{2} \int_{t_{0}}^{t} \frac{1}{\sqrt{t-\tau}}\left(\frac{D}{D \tau} \vec{V}-\frac{\partial}{\partial \tau} \vec{V}_{p}\right) d \tau+\frac{\pi}{6}\left(\rho_{p}-\rho\right) d_{p}^{3} \vec{g}+\vec{F}_{b}
\end{aligned}
$$


where, subscript $p$ stands for nanoparticle, $d_{p}$ is the diameter of a nanoparticle, $\rho_{p}$ is the density of nanoparticles and $\vec{V}_{p}$ is the velocity vector for nanoparticles. In this equation, the left hand side represents the inertial force of the particle. The right hand side of the equation comprises a number of forces. The first term represents the steady-state Stokes' drag force. The second term accounts for the non-viscous Froude-Krylov force, which is due to the pressure gradient in an unsteady flow field. The third term is the unsteady force due to the added (or virtual) mass effect, which can be interpreted as the force required for accelerating a volume of fluid, surrounding a particle. The fourth term indicates the unsteady Basset viscous force originating from the acceleration of a particle in the fluid media and subsequent lagging in the development of the associated boundary layer due to any change in relative velocity. The fifth term represents the non-viscous buoyancy force due to difference in density of the particle and that of the fluid. Finally, the last term on the right hand side of the equation is for other forces acting on the particle in the fluid such as the Brownian force.

Due to minute size of the particles, some other forces of hydrodynamic origin are not included in equation (6). These mainly include the Saffman lift force [13] produced by rotation of the particle caused by the velocity gradient in the viscous layers. In addition, the Faxen viscous force, which arises in the non-uniform flow field [12], is also neglected.

For sub-micrometre particles, the effects of Brownian motion can also be included. This is envisaged in the equation as $\vec{F}_{b}$. The components of the Brownian force are modelled as independent random white noise processes with a Gaussian distribution, having spectral intensities of $S_{n, i j}$ given by Li and Ahmadi [14] as:

$$
S_{n, i j}=\frac{216 \eta k_{B} T}{\pi^{2} \rho_{p}^{2} d_{p}^{5} C_{c}} \delta_{i j}
$$

in which, $k_{B}$ is the Boltzmann constant considered to be $1.38 \times 10^{-23} \mathrm{~J} / \mathrm{K}$ and $C_{c}$ is the Cunningham factor given by:

$$
C_{c}=1+\frac{2 \lambda}{d_{p}}\left[1.257+0.4 \exp \left(-1.1 \frac{d_{p}}{2 \lambda}\right)\right]
$$




\subsection{Cavitation model}

The lubricant is expected to cavitate in the diverging wedge of the conjunction (at the outlet of the contact). Various methods exist to treat the cavitation phenomenon in numerical models. However, the majority impose certain predefined conditions at the points of lubricant film rupture and subsequent reformation. Shahmohamadi et al [15-17] have shown that the cavitation phenomenon in tribological contacts can be treated in a more accurate manner, using a two-phase flow model which predicts the occurrence of cavitation inherently without the need for imposing any predefined boundary conditions. For this purpose, the cavitation model proposed by Singhal et al [18] is used, where the growth of cavities are confined in a gap of finite size. The transport equation for the vapour mass fraction is expressed as:

$$
\frac{\partial}{\partial t}\left(\rho f_{v}\right)+\vec{\nabla} \cdot\left(\rho f_{v} \vec{V}_{v}\right)=\vec{\nabla} \cdot\left(\Gamma \vec{\nabla} f_{v}\right)+R_{e}-R_{c}
$$

where, $\Gamma$ is the diffusion coefficient and $f_{v}$ is the vapour mass fraction. In addition, $R_{e}$ and $R_{c}$ are the source terms accounting for the rates of generation and condensation of vapour phase, respectively. Singhal et al [18] defined these phase change rates based on the generalised Rayleigh-Plesset equation as:

$$
\begin{array}{ll}
R_{e}=C_{e} \frac{V_{c h}}{S} \rho_{l} \rho_{v} \sqrt{\frac{2\left(p_{\text {sat }}-p\right)}{3 \rho_{l}}}\left(1-f_{v}\right), & \text { for } \quad p \leq p_{\text {sat }} \\
R_{c}=C_{c} \frac{V_{c h}}{S} \rho_{l} \rho_{v} \sqrt{\frac{2\left(p-p_{\text {sat }}\right)}{3 \rho_{l}}} f_{v}, & \text { for } \quad p>p_{\text {sat }}
\end{array}
$$

where, subscripts $l$ and $v$ refer to liquid and vapour phases, $p_{\text {sat }}$ is the saturation pressure, $V_{c h}$ is the characteristic velocity obtained as the local relative velocity between the liquid and the vapour phases. Since it is assumed that there are no dissolved gases in the lubricant at the start, the bubble pressure and vapour saturation pressure at any given temperature are considered to be the same. The empirical constants $C_{e}$ and $C_{c}$ are specified by Singhal et al [18] to be 0.02 and 0.01 respectively. 


\subsection{Lubricant rheology}

Two most important rheological properties of the lubricant; density and viscosity are functions of the operating temperature and pressure. Therefore, these need to be evaluated at the operating conditions.

For variations of density with pressure a modified version of the original model of Dowson and Higginson [19] by Yang et al [20] is used to take into account the effect of both pressure and temperature as:

$\rho=\rho_{0}\left(1+\frac{6.0 \times 10^{-10}\left(p-p_{\text {atm }}\right)}{1+1.7 \times 10^{-9}\left(p-p_{\text {atm }}\right)}\right)\left[1-0.65 \times 10^{-3}\left(T-T_{0}\right)\right]$

where, $p_{a t m}$ is the atmospheric pressure and $\rho_{0}$ and $T_{0}$ are the reference density and temperature respectively.

To take into account the variations of lubricant viscosity with pressure and temperature, the modified version of the Roelands' equation [21] by Houpert [22] is used:

$\eta=\eta_{0} \exp \left\{\left(\ln \left(\eta_{0}\right)+9.67\right)\left[\left(\frac{T+135}{T_{0}+135}\right)^{-S_{0}}\left(1+\frac{p-p_{a t m}}{1.98 \times 10^{8}}\right)^{Z}-1\right]\right\}$

where, $\eta_{0}$ is the lubricant dynamic viscosity at atmospheric pressure and reference temperature $T_{0} . Z$ and $S_{0}$ are given by [10]:

$Z=\frac{1.96 \times 10^{8} \alpha_{0}}{\ln \left(\eta_{0}\right)+9.67} \quad$ and $\quad S_{0}=\frac{\beta_{0}\left(T_{0}+135\right)}{\ln \left(\eta_{0}\right)+9.67}$

in which, $\alpha_{0}$ and $\beta_{0}$ are constants.

Details of the lubricant rheological properties are listed in Table 1.

\subsection{Asperity contact model}

In the mixed regime of lubrication, it is postulated that the instantaneous applied contact load is carried by a combination of lubricant hydrodynamic reaction and any direct asperity contact forces. Thus:

$F=\iint p d A+\iint \frac{16 \sqrt{2}}{15} \pi(\xi \beta \sigma)^{2} \sqrt{\sigma / \beta} E^{\prime} F_{5 / 2}\left(\lambda_{s}\right) d A$ 
where, the first term on the right hand side of the equation is the generated hydrodynamic reaction obtained through integration of the hydrodynamic pressure distribution, whilst the second term represents the normal reaction at the tip contact of an opposing asperity pair, calculated according to Greenwood and Tripp [23]. This term was originally developed for fairly smooth surfaces with a Gaussian distribution of asperity heights. Various rather laborious models exist which extend the application of the Greenwood and Tripp model for non-Gaussian surfaces such as those by Leighton et al [24, 25] for elastic interaction of asperities, Kogut and Etsion [26] for elasto-plastic adhesive dry contact and Chong et al [27] for wet asperities subjected to elastic deformation and adhesion.

Table 2 shows that roughness of the surfaces in the current study does not completely conform to a Gaussian distribution and hence ideally a more accurate model needs to be developed. However, for the sake of simplicity and to avoid further complexity, the conventional Greenwood and Trip model is adopted for the current study.

The statistical function $F_{5 / 2}\left(\lambda_{s}\right)$ takes into account the probability of asperity contacts at any given nominal gap between the two surfaces and is based on their Gaussian (normal) distribution. This is originally defined by Greenwood and Tripp [23] through the following statistical relationship:

$$
F_{5 / 2}(\lambda)=\int_{\lambda_{s}}^{\infty}\left(s-\lambda_{s}\right)^{5 / 2} \phi^{*}(s) d s
$$

where, $\phi^{*}$ is the standardised height distribution and $\lambda_{s}=h / \sigma$ is the film ratio. For further information on details of surface analysis the reader is referred to [24]. For a Gaussian distribution it is shown in [28] that a polynomial-fit function can be used to approximate the statistical integration as:

$$
F_{5 / 2}\left(\lambda_{s}\right)=\left\{\begin{array}{lr}
-0.004 \lambda_{s}^{5}+0.057 \lambda_{s}{ }^{4}-0.296 \lambda_{s}^{3}+0.784 \lambda_{s}{ }^{2}-1.078 \lambda_{s}+0.617 ; & \text { for } \lambda_{s} \leq \lambda_{c r} \\
0 ; & \text { for } \lambda_{s}>\lambda_{c r}
\end{array}\right.
$$

where, $\lambda_{c r} \cong 3$ is the critical value of film ratio, specifying the onset of mixed regime of lubrication.

\subsection{Conjunctional friction}

The total friction under mixed regime of lubrication is obtained as [10]: 
$f_{t}=\iint \bar{\tau}_{i j} d A+\pi(\xi \beta \sigma)^{2} \iint\left[\tau_{0} \pi F_{2}\left(\lambda_{s}\right)+\varsigma 16 \sqrt{2} / 15 \sqrt{\sigma / \beta} E^{\prime} F_{5 / 2}\left(\lambda_{s}\right)\right] d A$

where, the first integral represents the viscous component of friction created by bulk lubricant rheology and the second integral takes into account the boundary component of friction composed of direct dry asperity contact as well as the non-Newtonian shear of pockets of lubricant entrapped between the asperity summits. In the equation above, $\varsigma$ is the pressure coefficient for boundary shear strength of asperities. This is obtained using Lateral Force Microscopy (LFM) as described by Buenviaje et al [29] and Styles et al [30]. The limiting Eyring shear stress [31] of the lubricant used is $\tau_{0}=2 \mathrm{MPa}$ as is the case for the majority of engine lubricants. In addition, the statistical function $F_{2}\left(\lambda_{s}\right)$ is given in polynomial form as [28]:

$$
F_{2}\left(\lambda_{s}\right)= \begin{cases}-0.0018 \lambda_{s}{ }^{5}+0.0281 \lambda_{s}{ }^{4}-0.1728 \lambda_{s}^{3}+0.5258 \lambda_{s}^{2}-0.8043 \lambda_{s}+0.5003 ; & \text { for } \lambda_{s} \leq \lambda_{c r} \\ 0 ; & \text { for } \lambda_{s}>\lambda_{c r}\end{cases}
$$

\subsection{Flow and thermal boundary conditions}

The thermal and flow boundary conditions are shown in Figure 3. Continuity of heat flux and compatibility of temperature at the lubricant-solid interfaces are assured. As the lubricant is entrained into the contact, its temperature rises due to shear as well as heat generated because of any asperity contact. The generated heat is transferred away through mass convection by the passing lubricant, as well as through conduction to the adjacent contacting solid surfaces. In the conducted experiments with the pin-on-disc tribometer all the extremities of both the pin and the disc are exposed to the ambient air, apart from the bottom surface of the disc, resting upon its mounting. Therefore, it is expected that convection cooling would take place at all the exposed surfaces to the ambient. All thermal and lubricant flow boundary conditions are shown in figure 3. 


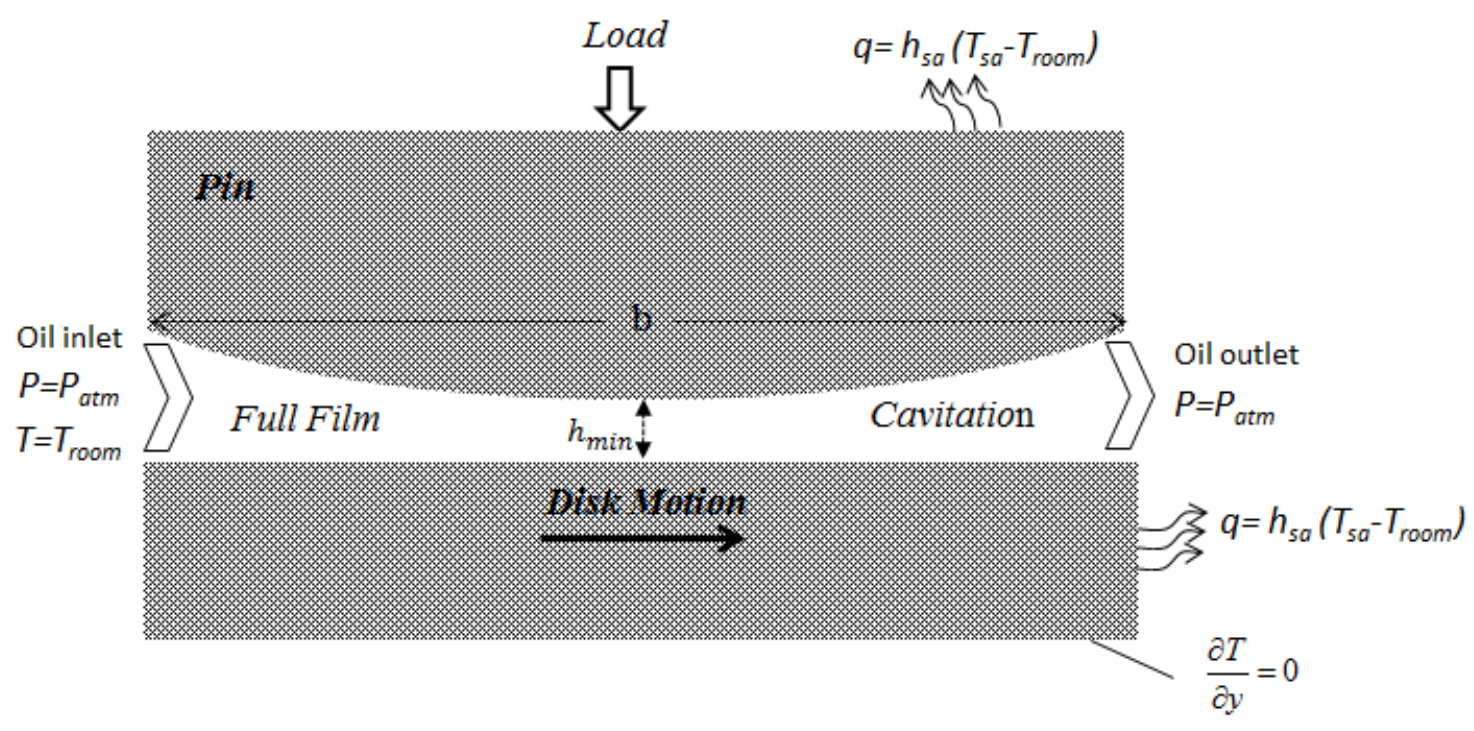

Figure 3: Schematic representation of the lubricant flow and thermal boundary conditions

\section{Solution Procedure}

A 2-dimensional thermal flow dynamics model is developed using the Ansys Fluent 14.5. For the grid generation, the pre-processor Ansys Design Modeller and Meshing is used. The thinness of the lubricant film necessitates the use of quadrilateral grid volumes. The entire flow domain is meshed using 300,000 quadrilateral mesh volumes. Preliminary runs are conducted to ensure mesh-independency of all subsequent analyses. Lubricant flow is as laminar because of low Reynolds number, typical of thin-film tribological conjunctions. The cavitation vaporisation pressure of the lubricant is set to the atmospheric pressure at 101.3 $\mathrm{kPa}$. Atmospheric inlet and outlet pressure boundary conditions are used for the leading and trailing edges of the pin-on-disc contact. It is also assumed that the inlet of the contact remains fully flooded as in the experiments an ample supply of lubricant is made available as previously described. The pressure-based mixture model of Manninen et al [32] is chosen for the present flow analysis. The velocity-pressure coupling was treated using the Semi-implicit Method for Pressure-linked Equations (SIMPLE) algorithm, whilst a second-order upwind scheme is used for the solution of the momenta in order to reduce the discretisation-induced errors of calculations. The time step of simulation is in the order of $10^{-3}$. Since the flow is not dominated by buoyancy (i.e. low Rayleigh number), this time step is well within the required limit. 


\section{Results and Discussion}

Comparison of coefficients of friction for the base oil and that containing nanoparticles at two different disc rotational speeds of $600 \mathrm{rpm}$ and $1000 \mathrm{rpm}$ is obtained experimentally and also through numerical analysis as shown in figure 4. The coefficient of friction rises with increased disc speed because of a higher shear rate, which is an expected outcome. With the presence of nanoparticles the coefficient of friction is higher than that with the base oil at both sliding speeds. The reason for this is that a thicker film is formed at lower contact temperature, with higher viscosity. The dominant viscous shear stress in the contact is $\frac{U \eta}{h}$, therefore the increase in viscosity at lower contact temperature because of nanoparticles is the predominant reason for reduced viscous shear, thus friction compared with the base oil alone. Therefore, there is a higher heat transfer due to the presence of nanoparticles.

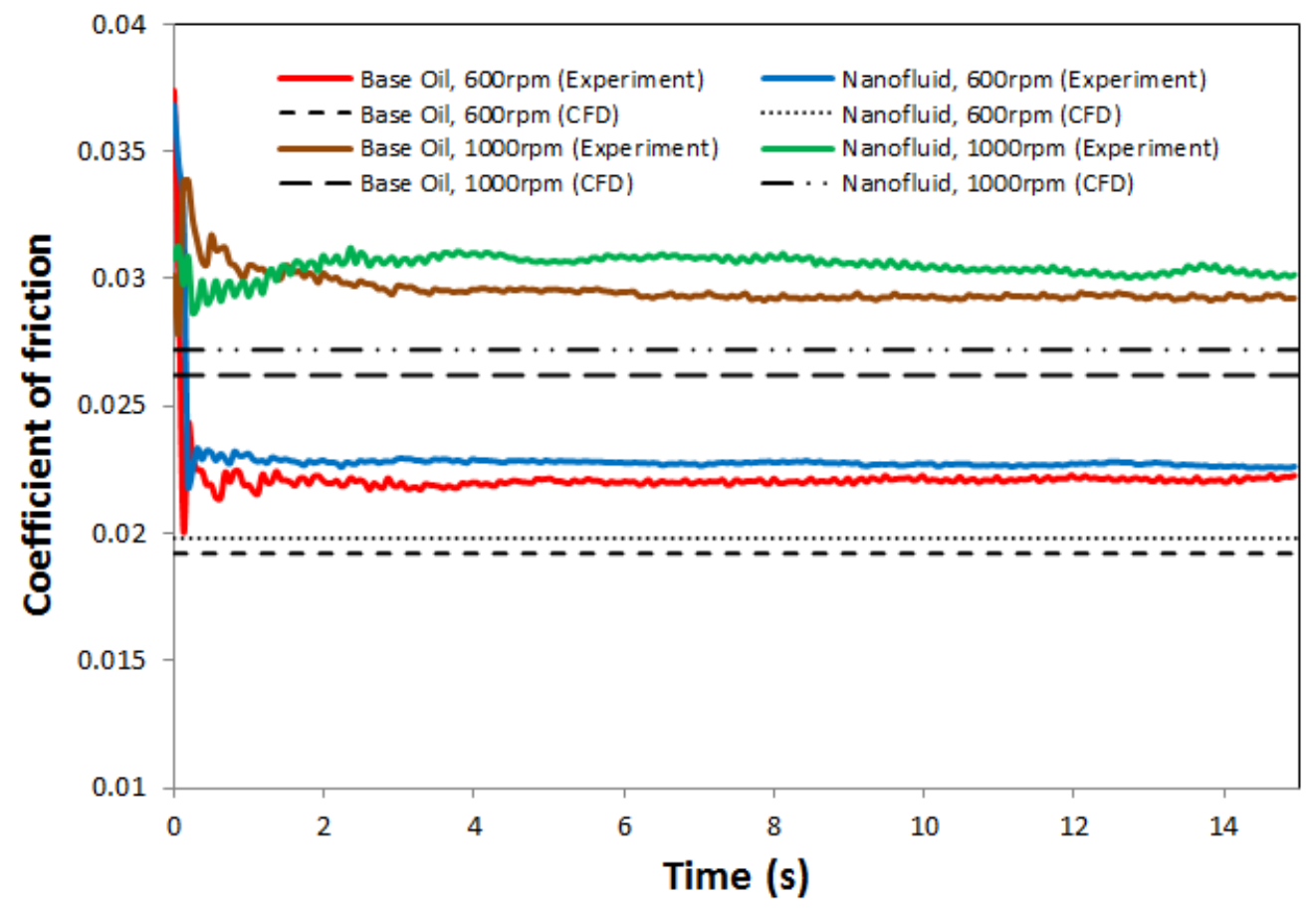

Figure 4: Comparison of coefficient of friction for base oil alone and that with nanoparticles: measured and predicted

Figure 5 shows constructed Stribeck curves for both the base oil and that containing nanoparticles at two different applied loads. The coefficients of friction for both applied loads of 5 and $10 \mathrm{~N}$ dramatically decrease from 0.11 and 0.2 to 0.01 and 0.02 , when the sliding speed is increased up to 0.6 and $1.4 \mathrm{~m} / \mathrm{s}$ respectively, since the flow operates in the mixed regime of lubrication. At higher speeds and lower loads, due to the converging-diverging 
profile of the slider, prevalence of a hydrodynamic film is expected and as a result the coefficient of friction remains quite low. Nevertheless, in hydrodynamic regime of lubrication and at a constant load, viscous shear increases proportially with the sliding speed as it can be observed from figure 5 . Therefore, under hydrodynamic regime of lubrication the coefficient of friction remains almost the same with or without the presence of nanoparticles in the base oil. The results in figures 4 and 5 show that the main effect of nanoparticles is under mixed regime of lubrication with occurece of thinner films.

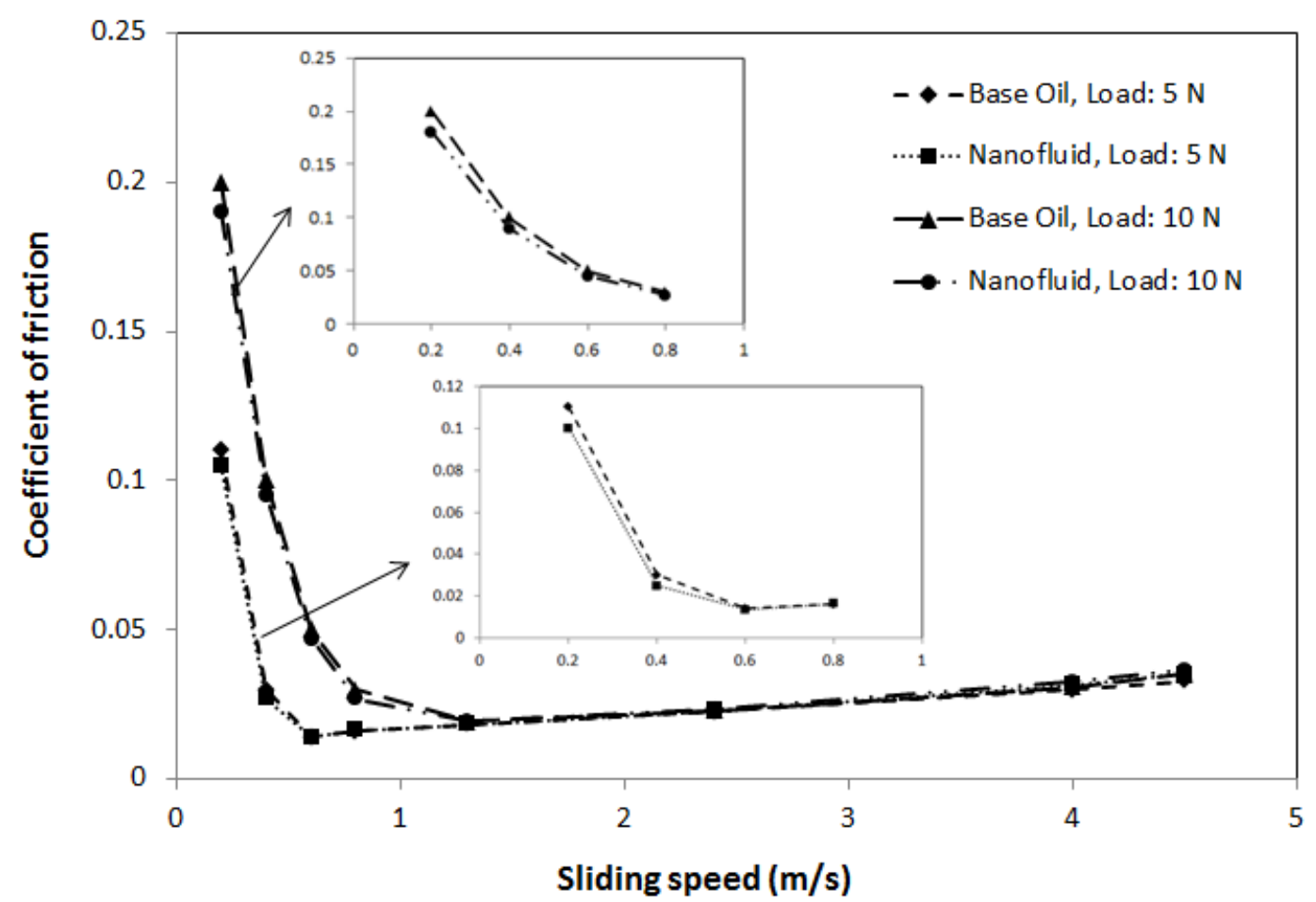

Figure 5: Stribeck curves for base oil and nanofluid at two different loads

Typical pressure distribution in the pin-on-disc conjunction due to direct asperity contact is shown in Figure 6. This is for the case of sliding speed of $0.4 \mathrm{~m} / \mathrm{s}$ (equivalent to rotational speed of $100 \mathrm{rpm}$ ) and an applied contact load of $10 \mathrm{~N}$. The calculated Stribeck film ratio in this case is $\lambda_{s}=1.5$ (well within a mixed regime of lubrication). 


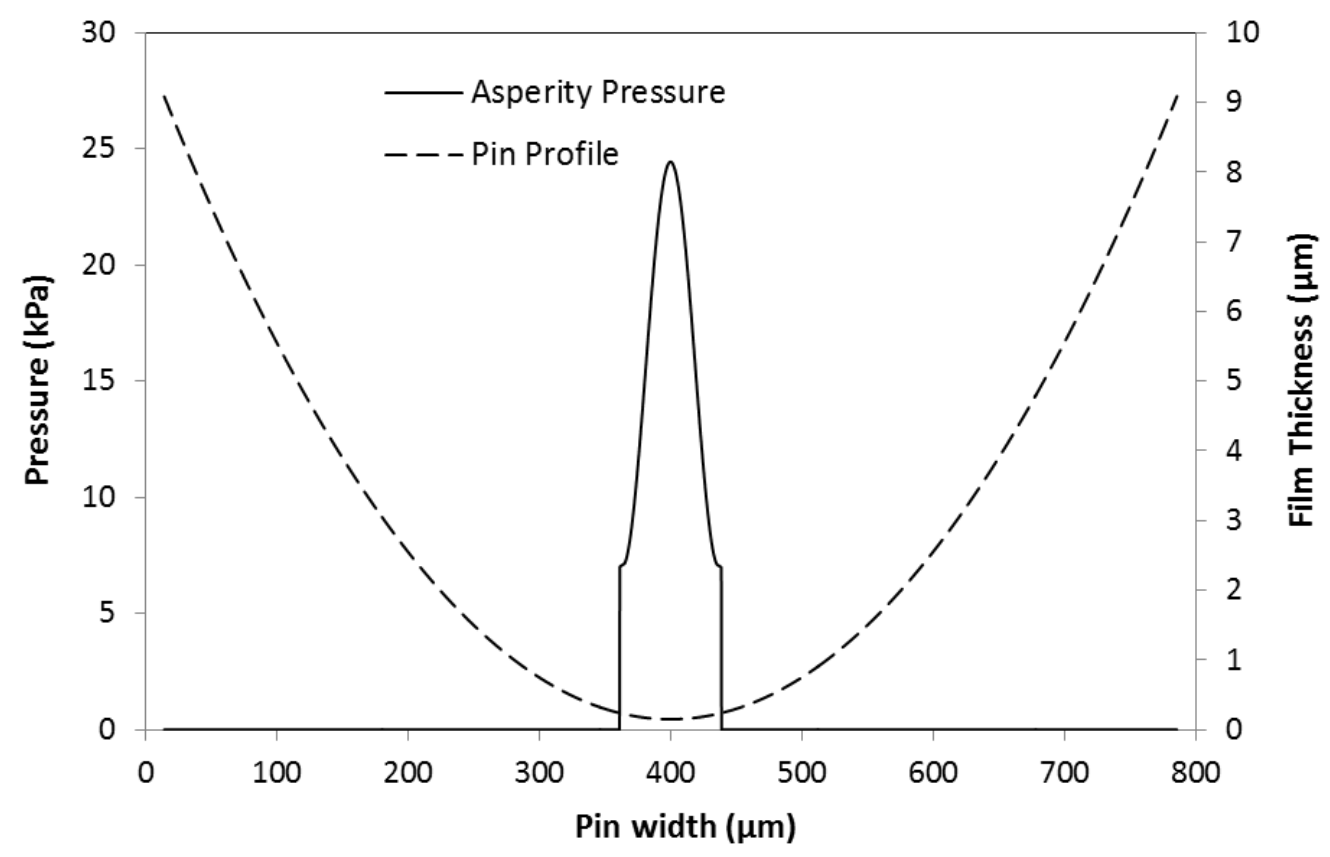

Figure 6: Asperity pressure distribution along the contact for $\lambda_{\mathrm{s}}=1.5$

Average film temperature for the base oil and that with nanoparticles at different speeds is presented in figure 7 . The average temperature in the case of the latter is lower. This is because of the much higher thermal conductivity of the solid nanoparticles, resulting in a lower contact operating temperature. Consequently, the nano-lubricant retains a higher viscosity than the base oil alone. This also accounts for the retention of a thicker lubricant film with nanoparticles (figure 8).

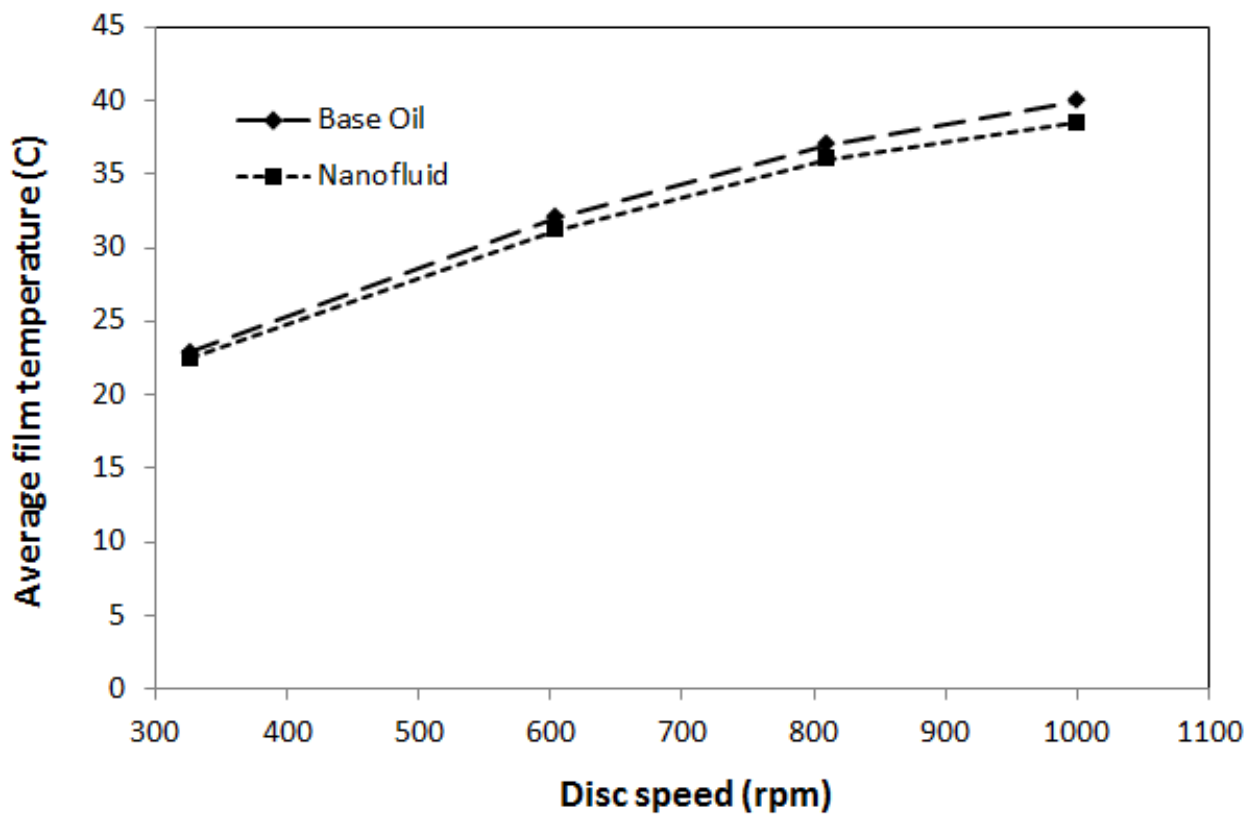


Figure 7: Average temperature of base oil and that with nanoparticles at different speeds

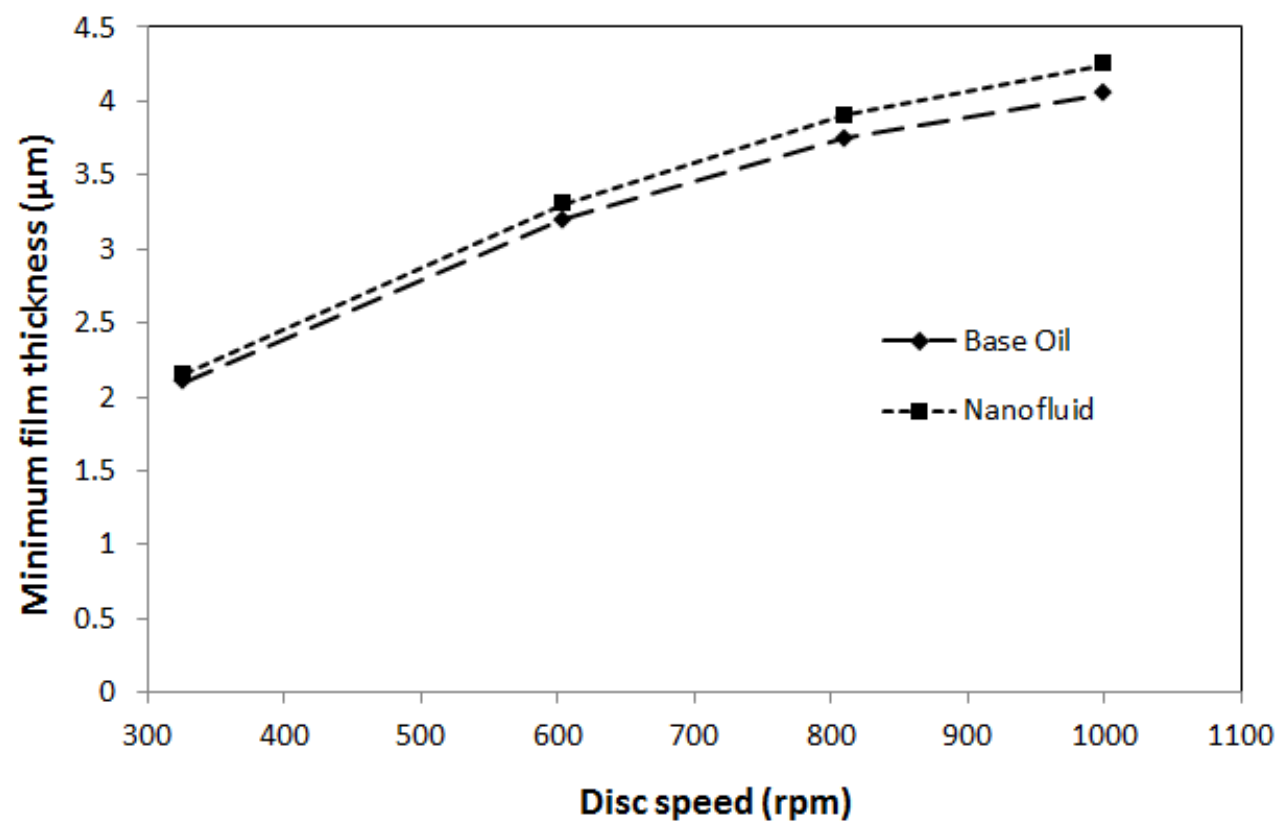

Figure 8: Minimum film thickness for the base oil and that with nanoparticles at different disc speeds

Figure 9 shows the pressure distribution along the pin width for both the base oil and that containing nanoparticles. It can be seen that the lubricant film ruptures earlier in the case of the lubricant without nano-lubricant. A small cavitation region in the nanoparticle flow indicates that vapour volume fraction is quite low in this case. This is another reason for the formation of thicker films with presence of nanoparticles as is observed in the results of figure 8 . 


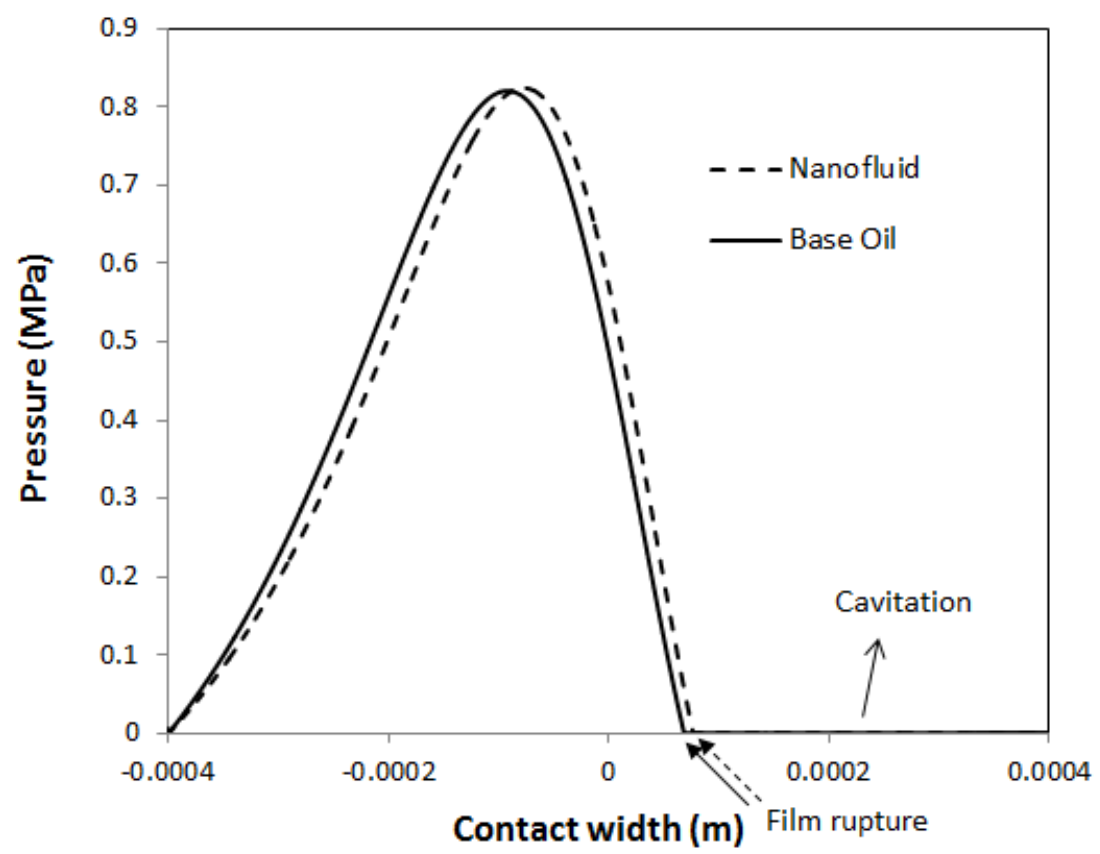

Figure 9: Pressure distribution along the pin width

\section{Conclusions}

A combined experimental and numerical analysis of the tribological performance of a polyalphaolefin PAO6 base oil with $0.02 \%$ volume fraction carbon nanoparticle is carried out. The detailed numerical analysis comprises simultaneous solution of Navier-Stokes equations with discrete dispersed phase represented by Lagrangian dynamics, energy equation, modified Rayleigh-Plesset equations for finite gap conjunctions and vapour transport equation to take into account any effect of cavitated flow. A broad range of operating conditions from boundary to mixed and through to hydrodynamic regimes of lubrication, by the inclusion of asperity interactions using Greenwood and Tripp model. The analysis represents the most fundamental modelling approach for nano-lubricant action hitherto in reported in literature. Experimental work is carried out with a temperature controlled fully instrumented pin-on-disc tribometer with precision lubricant feed rate.

Stribeck curves are obtained both experimentally and predicted numerically under a broad range of operations, indicating the effectiveness of nanoparticles in enhanced heat transfer from the contact domain, thus retaining a higher viscosity thicker lubricant film with reduced friction. Furthermore, a small cavitation region is formed at the contact exit, which indicates low volume fraction of cavities, insufficient to impede heat transfer from the lubricant film to the adjacent solid boundaries. Numerical predictions conform well to the experimental measurements. 
The main findings of the paper are:

- No significant difference in frictional performance of a PAO6 base oil with $0.02 \%$ concentration of carbon nanoparticles is noted.

- The effect of nanoparticles is more pertinent in mixed and boundary regimes of lubrication, where slightly lower coefficient of friction is achieved due to reduced contact temperature. Therefore, a thicker lubricant film is attained. It is shown that this is due to the ability of nanoparticles in removing the heat away from the contact.

- Existence of nanoparticles seems to produce a shift in the loci of the maximum pressure and lubricant film rupture point, with implication on reducing the extent of lubricated contact through cavitation.

The approach expounded in this paper can be further improved both in experimentation and numerical predictions. In the case of the former, it would be necessary to accurately determine the Eyring and limiting shear stresses of the base oil and that with the inclusion of nanoparticles under the conditions experienced in the pin-on-disc contact, rather than a nominal value measured through standard viscometry, generally of low to medium shear at relatively low pressures. In the case of the latter a non-Gaussian, surface specific boundary friction model in line with the recently reported in [25] would represent prediction of boundary friction more faithfully. Inter-particle forces and those between the particles and atoms of the solid contacting surfaces would play a role in their in situ distribution in transit through the contact. These forces would include electrostatic repulsion as well as long range van der Waal interactions. They also become dominant with reduced contact load, thinness of lubricant film (mixed and boundary regimes of lubrication) as well as smoothness of contacting surfaces [10,33]. The aggregation of nanoparticles and the attachment/adsorption of particles to the solid boundaries is a function of these forces, as well as the Faxen viscous force in their detachment, also not included in this preliminary analysis. Inclusion of these forces is not seen as critical at the contact loads investigated here. Nevertheless, a couplestress fluid model may also be considered to address the complexity of fluid flow of the nanolubricants caused e.g. by polar effects and the rotation of the nanoparticles. All these additions constitute the future direction of this research.

\section{Acknowledgements}

The authors would like to acknowledge the financial support of Lloyds Register Foundation (LRF) to the International Cavitation Research Institute, under whose auspices the current 
research is carried out. The financial support of the Innovate UK in the use of nanoparticles is also acknowledged.

\section{References}

[1]- Greenberg, R., Halperin, G., Etsion, I. and Tenne, R., "The effect of WS2 nanoparticles on friction reduction in various lubrication regimes", Tribology Letters, 2004, 17(2), pp.179186

[2]- Wu, Y.Y., Tsui, W.C. and Liu, T.C., "Experimental analysis of tribological properties of lubricating oils with nanoparticle additives”, Wear, 2007, 262(7), pp.819-825

[3]- Battez, A.H., Viesca, J.L., González, R., Blanco, D., Asedegbega, E. and Osorio, A., "Friction reduction properties of a $\mathrm{CuO}$ nanolubricant used as lubricant for a NiCrBSi coating”, Wear, 2010, 268(1), pp.325-328

[4]- Lee, J., Cho, S., Hwang, Y., Lee, C. and Kim, S.H., "Enhancement of lubrication properties of nano-oil by controlling the amount of fullerene nanoparticle additives", Tribology Letters, 2007, 28(2), pp.203-208

[5]- Ku, B.C., Han, Y.C., Lee, J.E., Lee, J.K., Park, S.H. and Hwang, Y.J., “Tribological effects of fullerene (C60) nanoparticles added in mineral lubricants according to its viscosity”, International Journal of Precision Engineering and Manufacturing, 2010, 11(4), pp.607-611.

[6]- Lee, C.G., Hwang, Y.J., Choi, Y.M., Lee, J.K., Choi, C. and Oh, J.M., “A study on the tribological characteristics of graphite nano lubricants”, International Journal of Precision Engineering and Manufacturing, 2009, 10(1), pp.85-90

[7]- Wan, Q., Jin, Y., Sun, P. and Ding, Y., “Tribological Behaviour of a Lubricant Oil Containing Boron Nitride Nanoparticles”, Procedia Engineering, 2015, 102, pp. 1038-1045.

[8]- Godson, L., Raja, B., Lal, D.M. and Wongwises, S., "Enhancement of heat transfer using nanofluids - An overview, Renewable and Sustainable Energy Reviews”, 2010, 14, pp. 629-641

[9]- Kamyar, A., Saidur, R. and Hasanuzzaman, M., “Application of computational fluid dynamics (CFD) for nanofluids”, International Journal of Heat and Mass Transfer, 2012, 55(15), pp.4104-4115

[10]- Gohar, R. and Rahnejat, H., "Fundamentals of Tribology”, Imperial College Press, London, 2008.

[11]- White, F.M., “Viscous Fluid Flow”, McGraw-Hill, 2nd Edition, New York, 1991.

[12]- Crowe, C.T., Schwarzkopf, J.D., Sommerfeld, M. and Tsuji, Y., ”Multiphase flows with droplets and particles”, CRC press, 2011. 
[13]- Saffman, P.G.T., "The lift on a small sphere in a slow shear flow, Journal of Fluid Mechanics, 1965, 22(02), pp. 385-400.

[14]- Li, A. and Ahmadi, G., "Dispersion and Deposition of Spherical Particles from Point Sources in a Turbulent Channel Flow", Aerosol Science and Technology, 1992, 16, pp. 209226.

[15]- Shahmohamadi, H., Rahmani, R., Rahnejat, H., Garner, C.P. and King, PD., “ThermoMixed Hydrodynamics of Piston Compression Ring Conjunction”, Tribology letters, 2013, 51, pp. 321-340.

[16]- Shahmohamadi, H., Mohammadpour, M., Rahmani, R., Rahnejat, H., Garner, C.P. and Howell-Smith, S., "On the boundary conditions in multi-phase flow through the piston ringcylinder liner conjunction”, Tribology International, 2015, 90, pp. 164-174.

[17]- Shahmohamadi, H., Rahmani, R., Rahnejat, H., Garner, C.P. and Dowson, D., "Big end bearing losses with thermal cavitation flow under cylinder deactivation”, Tribology Letters, 2015, 57, pp. 1-17.

[18] Singhal, A.K., Li H.Y., Athavale M.M. and Jiang Y., "Mathematical Basis and Validation of the Full Cavitation Model”, ASME FEDSM'01, New Orleans, Louisiana, 2001.

[19]- Dowson, D. and Higginson, G.R., “A Numerical Solution to the Elasto-Hydrodynamic Problem”, J. Mech. Eng. Sci., 1, 1959, pp. 6-15.

[20]- Yang, P., Cui, J., Jin, Z.M. and Dowson, D., “Transient elastohydrodynamic analysis of elliptical contacts; Part 2: thermal and Newtonian lubricant solution”, Proc. IMechE, Part J: J. Engineering Tribology, 2005, 219, pp. 187-200

[21]- Roelands, C.J.A., "Correlation aspects of the viscosity-temperature-pressure relationships of lubricating oils”, Druk: VRB, Kleine der A3-4, Groningen, 1966

[22]- Houpert, L., "New results of traction force calculations in elastohydrodynamic contacts”, Trans. ASME, J. Tribology., 1985, 107, pp. 241-248.

[23]- Greenwood, J.A. and Tripp, J.H., "The contact of two nominally flat rough surfaces", Proc IMechE, J. Mechanical Engineering Science, 1970-1971, 185, pp. 625-634.

[24]- Leighton, M., Morris, N., Rahmani, R. and Rahnejat, H., "Surface specific asperity model for prediction of friction in boundary and mixed regimes of lubrication, Meccanica, 2017, 52(1), pp. 21-33

[25]- Leighton, M., Morris, N., Gore, M., Rahmani, R., Rahnejat, H. and King, P.D., "Boundary interactions of rough non-Gaussian surfaces", Proc. IMechE, Part J: J. Engineering Tribology, 2016, 230(11), pp. 1359-70 
[26]- Kogut, L and Etsion, I., “Adhesion in elastic-plastic microcontact”, J. Colloid Interface Science, 2003, 261, pp. 372-378.

[27]- Chong, W.W., Teodorescu, M. and Rahnejat, H., "Nanoscale elastoplastic adhesion of wet asperities”, Proc. IMechE, Part J: J. Engineering Tribology, 2013, 227(9), pp. 996-1010.

[28]- Teodorescu, M., Balakrishnan, S. and Rahnejat, H., "Integrated tribological analysis within a multi-physics approach to system dynamics”, Tribology and Interface Engineering Series, 2005, 48, pp. 725-737.

[29]- Buenviaje, C.K., Ge, S.-R., Rafaillovich, M.H. and Overney, R.M., “Atomic force microscopy calibration methods for lateral force, elasticity, and viscosity”, Mater. Res. Soc. Symp. Proc., 1998, 522, pp. 187-192.

[30]- Styles, G., Rahmani, R., Rahnejat, H. and Fitzsimons, B., "In-cycle and life-time friction transience in piston ring-liner conjunction under mixed regime of lubrication”, Int. J. Engine Research., 2014, 15, pp. 862-876.

[31]- Eyring, H., "Viscosity, plasticity and diffusion as examples of reaction rates", J. Chem. Phys., 1926, 4, pp. 283-291.

[32]- Manninen, M., Taivassalo, V. and Kallio, S., "On the mixture model for multiphase flow, VTT Publications, 1996, 288, Technical Research Centre of Finland, Helsinki, Finland.

[33]- Al-Samieh, M. and Rahnejat, H., "Ultra-thin lubricating films under transient conditions”, J. Phys., D: Applied Physics, 2001, 34(17), 2610. 